\title{
Interactive Spatial Visualization of Aggregated Non-spatial Coronavirus Data
}

\author{
Rostislav Netek ${ }^{a}, *$, Jakub Konicek ${ }^{\text {a }}$ \\ ${ }^{a}$ Dept. of Geoinformatics, Palacký University Olomouc, Rostislav Netek - rostislav.netek@ upol.cz, Jakub Konicek - \\ jakub.konicek@upol.cz \\ * Corresponding author
}

Keywords: aggregator, social media, covid-19, visualisation

\begin{abstract}
:
The article describes the process of aggregation of media-based data about coronavirus pandemic in the Olomouc region, Czech Republic. Originally non-spatially located news from different sources and various platforms (government, social media, news portals) were automatically aggregated into a centralized database. The application "COVID-map" is an interactive web map solution which visualizes records from the database in a spatial way. COVIDmap has been developed within Ad hoc online hackathon as an academic project at the Department of Geoinformatics, Palacký University Olomouc, Czech Republic. Alongside spatially localized data, map application collects statistical data from official sources e.g. from the governmental crisis management office.

Our approach focuses on cartographical aspects of COVID-map solution. It respects current trends in both development of cartographic oriented outputs and web-based interactive map application. It is fully responsive. Originally, nonspatial data were aggregated, verified, geo-located, and finally visualized in the map environment. The layout combines three main parts: interactive map with spatial data; information panel with updated statistical data; and a sidebar with a filterable list of verified and geo-located news. The main cartographic method used for the visualisation is the choropleth map. It shows the distribution of positively tested people per 10000 inhabitants for each sub-region. In comparison with similar coronavirus visualisation made during the current pandemic, our solution respects fundamental cartographical rules.
\end{abstract}

Following cartographical topics are discussed in the article: the correct choice of cartographic method and its implementation; determination of a colour scale; recalculation statistics data into relative units by choropleths map method; design of map symbols and legend; searching for a suitable visualization of spatial and non-spatial sources into map layout; discussion on the suitability of using a clustering method.

The motivation for this project was both social responsibility and dissatisfaction with the wrong implementation of basic cartographic methods in a variety of alternative solutions. The impact was immediate. Within a few days after the launch, tens of thousands users per day visited the COVID-map. It has been published by regional and national media. The COVID-map solution could be considered as a suitable ad-hoc implementation of the correctly used cartographical method on the example of coronavirus pandemic. The application "COVID-map" is available online at URL: https://gis.upol.cz/covid/mapa/. 


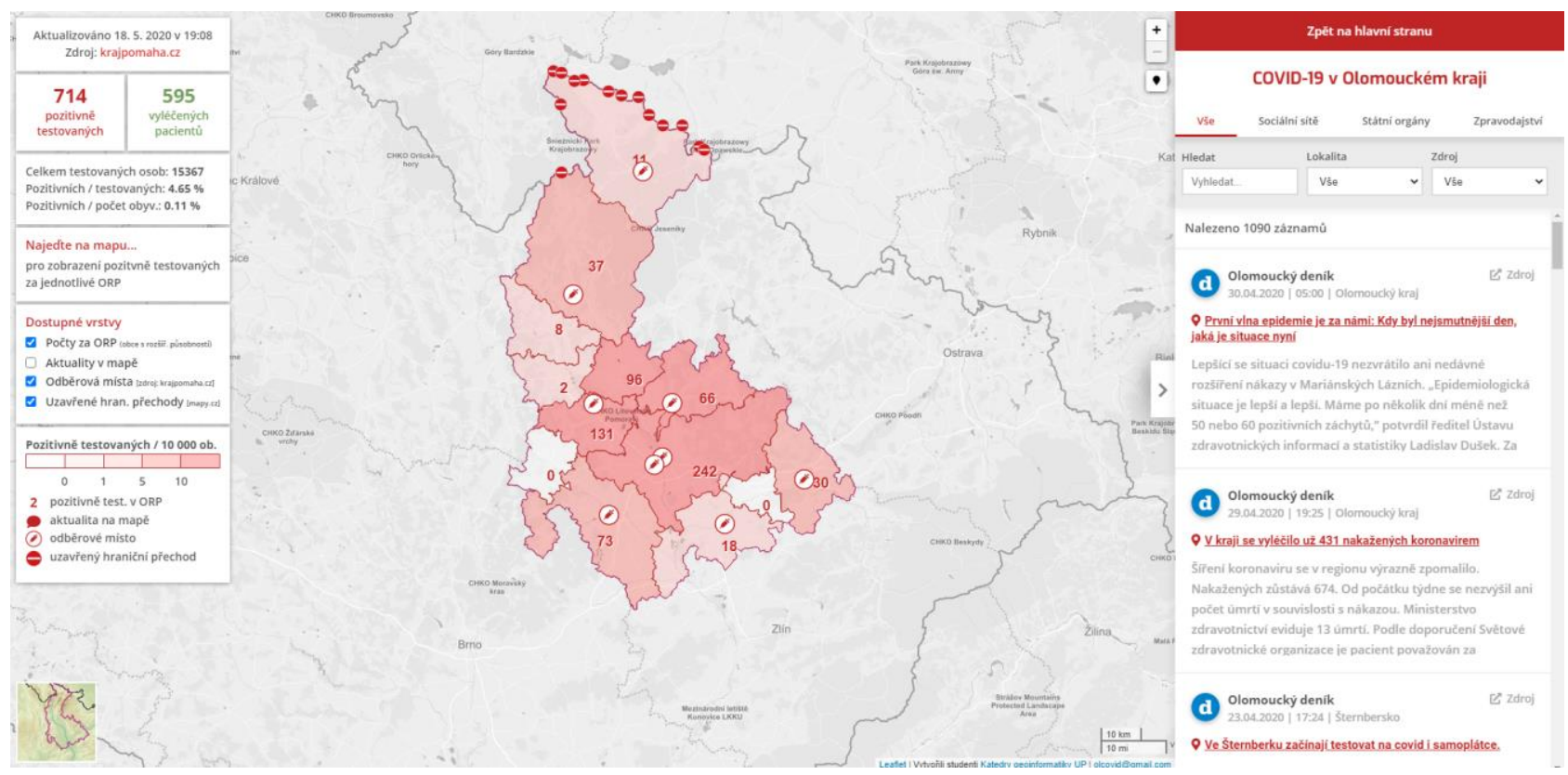

Figure 1. COVID-map interface combines statistical, multimedia and spatial data

\section{Acknowledgements}

This paper was supported by the project "Spatial Synthesis Based in Advanced Geocomputation Methods" (reg. num. 18-05432S) of Czech Science Foundation. 